\title{
Endovascular Reconstruction for Treatment of Vertebrobasilar Dolichoectasia: Long-Term Outcomes
}

\author{
X. Wu, Y. Xu, B. Hong, W.-Y. Zhao, Q.-H. Huang, and J.-M. Liu
}

\begin{abstract}
BACKGROUND AND PURPOSE: VBD can trigger various clinical symptoms, especially ischemic stroke in the posterior circulation, but there is no effective treatment for their prevention. We aimed to validate the feasibility of coil-assisted stent reconstruction in the vascular lumen for the treatment of VBD and to evaluate its long-term effectiveness in preventing ischemic events.
\end{abstract}

MATERIALS AND METHODS: Clinical and imaging data of 9 patients with unruptured VBD were reviewed retrospectively. Depending on the length and diameter of the diseased target vessel, multiple LEO and/or Solitaire stents were implanted, assisted by coils.

RESULTS: Stent reconstruction in the vascular lumen was successful in all patients. Procedure-related complications occurred in 1 patient who developed brain stem and cerebellar infarction 8 days after endovascular treatment and died in 4 months. Coil embolization of the nondominant side of the vertebral artery was performed 1 month after the operation in 1 patient who developed partial brain stem and cerebellar infarction leading to hemiplegia. The mean follow-up time of the 8 surviving patients was $20.75 \pm 6.90$ months. Of the 4 patients with dolichoectasia in the anterior circulation, 2 experienced ischemic events in the anterior circulation. Another patient had sudden death at home 26 months after the operation. The conditions of the remaining 5 patients were stable without deterioration, and in 4, DSA/ CTA/MRA suggested improved morphology of the vertebral artery compared with that before the operation.

CONCLUSIONS: Endovascular reconstruction with coil-assisted stent placement or stent placement alone in the vascular lumen for the treatment of VBD is technically feasible and can prevent ischemic events in the territory of stented vessels compared with the natural course, though further studies in larger samples are needed.

ABBREVIATIONS: IADE = intracranial arterial dolichoectasia; $\mathrm{mRS}=$ modified Rankin Scale; $\mathrm{PCA}=$ posterior cerebral artery; $\mathrm{VA}=$ vertebral artery; $\mathrm{VBD}=$ vertebrobasilar dolichoectasia

ADE is a dilative arteriopathy characterized by local dolichoectasia of the intracranial arteries. The incidence of IADE is approximately $0.06 \%-5.8 \%,{ }^{1-6}$ of which VBD accounts for approximately $78 \% .^{7}$ Although the incidence of VBD is not high, it may

Received February 23, 2012; accepted after revision May 29.

From the Department of Neurosurgery, Changhai Hospital, Second Military Medical University, Shanghai, China.

Xi Wu performed data analysis, documentation, and manuscript writing. Yi Xu, Bo Hong, Wen-Yuan Zhao, and Qing-Hai Huang performed cases. Qing-Hai Huang was also responsible for manuscript editing and data analysis. Jian-Min Liu was responsible for performing operations, data analysis, and manuscript writing.

There were no conflicts no interest.

This work was supported by the National Natural Science Foundation of China (grant No. 81171092 and grant No. 81171093), Rising-star Plan of Shanghai Science and Technology Committee (grant No. 11QA1408400), and the Second Military Medical University Science Fund for Young Scholars (X. Wu)

Please address correspondence to Jian-Min Liu, MD, or Qing-Hai Huang, MD, Department of Neurosurgery, Changhai Hospital, Second Military Medical University, 168 Changhai Rd, Shanghai, China; e-mail: chstroke@163.com or ocinhqh@163.com

- Indicates open access to non-subscribers at www.ajnr.org

http://dx.doi.org/10.3174/ajnr.A3248 trigger various clinical symptoms, especially ischemic stroke in the posterior circulation. ${ }^{6,8}$ A long-term follow-up study in 156 VBD cases showed that $39.7 \%$ (62/156) of patients died during the follow-up period. ${ }^{8}$ However, there is still a lack of an effective treatment for VBD. ${ }^{9}$ This study retrospectively reviewed the clinical and imaging data of 9 patients with VBD who were treated with coil-assisted stent reconstruction in the vascular lumen, in an attempt to explore the feasibility and long-term effectiveness of this technique in preventing ischemic events in stented vessels.

\section{MATERIALS AND METHODS}

This retrospective review included the clinical and imaging data of patients whose diagnosis was consistent with the CT and MR imaging diagnostic criteria for $\mathrm{VBD}^{10,11}$ and was confirmed by 2 specialists in cerebrovascular diseases. All patients received multiple overlapping stents alone or coil-assisted stent reconstruction in the vascular lumen for VBD in our center between January 31, 2004, and August 31, 2011. Informed consent was obtained from all patients and their relatives. The research protocol was ap- 

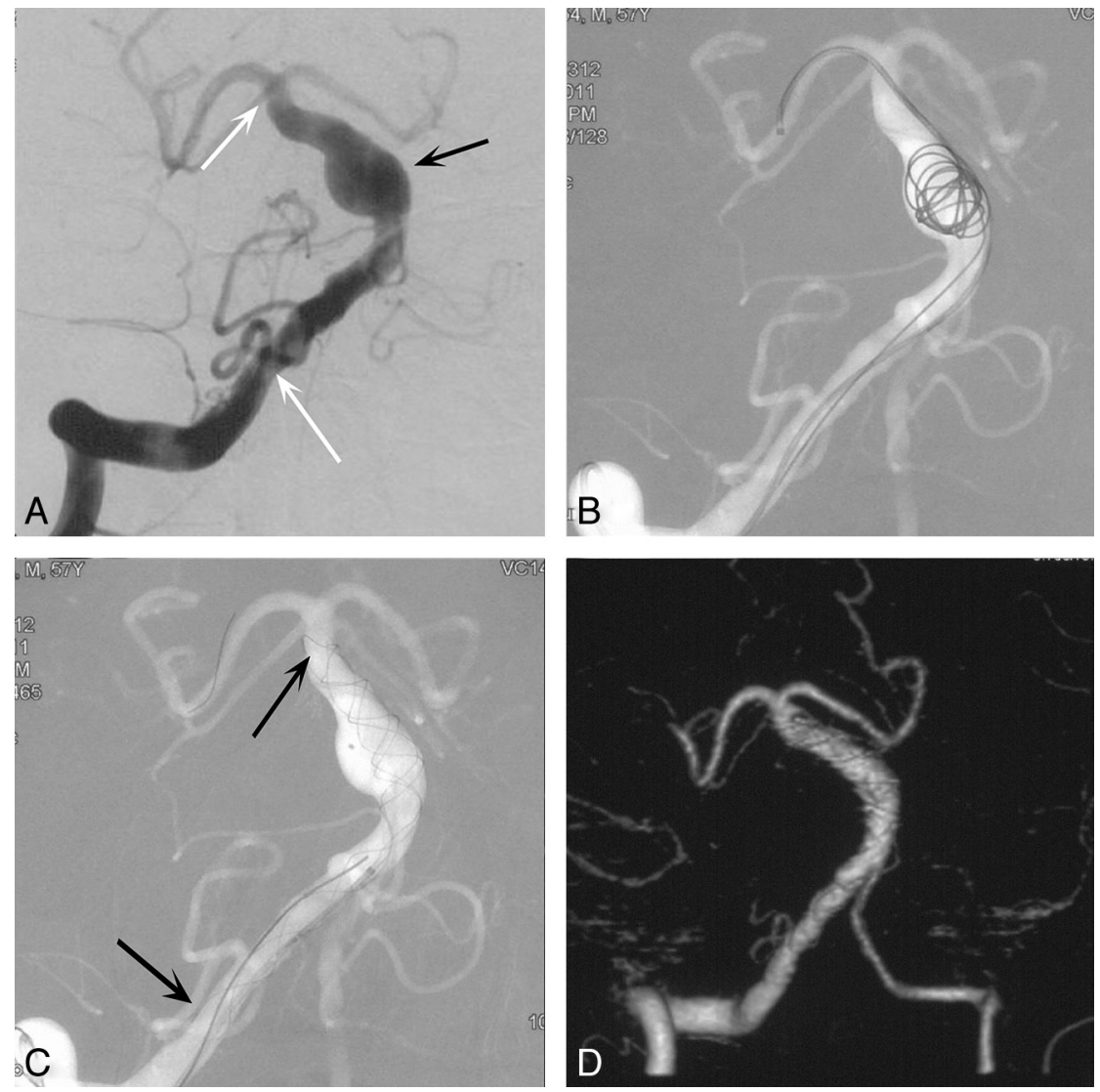

FIG 1. A, Anteroposterior position angiogram of the right vertebrobasilar artery depicts the VBD (white arrows) and a fusiform aneurysm (black arrow). B, Semideployed coil to assist 2 telescoping LEO stents deployed with the double-catheter technique. $C$, The coil is resheathed after stent deployment. $D$, The right vertebrobasilar artery lumen is smaller than that in $A$ (CT angiography follow-up 24 months). Note the disappearance of the fusiform aneurysm.

proved by the institutional review board of Changhai Hospital (Shanghai, China). In our center, stent reconstruction for VBD was indicated for: 1) patients whose primary symptom was stroke; 2) patients whose condition deteriorated gradually as indicated by clinical or imaging follow-up; and 3) patients with VBD-complicated acute dissection, aneurysmal arterial wall vesicles, or aneurysms.

The patients with IADE involving the carotid and vertebrobasilar arteries were separated into a subgroup. The natural course of IADE was further followed up after endovascular reconstruction for VBD.

\section{Treatment}

Anticoagulation and Antiplatelet Protocols. Dual antiplatelet agents ( $75 \mathrm{mg} /$ day of clopidogrel and $300 \mathrm{mg}$ /day of aspirin) were administered for 3 days before the procedure. All patients were maintained on aspirin and clopidogrel for 6 weeks, followed by indefinite continuation of aspirin, $100 \mathrm{mg} /$ day, alone.

Strategies and Endovascular Treatment. All patients underwent endovascular procedures under general anesthesia and received systemic heparinization after placement of the sheath. The activated clotting time was maintained at 2-3 times the baseline throughout the procedure. A 6F Envoy guide catheter (Cordis,
Miami Lakes, Florida) was navigated to the $\mathrm{C} 1 \sim 2$ level of the dominant side of the VA, as defined by Jeng and Yip. ${ }^{12}$ Depending on the length and diameter of the diseased target artery, multiple LEO self-expanding stents (Balt Extrusion, Montmorency, France) and Solitaire stents (ev3, Irvine, California) were implanted, and we ensured that the distal portion of the stent was placed in the normal portion of the distal target vessel. The lumen of the diseased artery was reconstructed by the overlapping technique, and we ensured that the proximal portion of the stent covered the proximal normal portion of the target artery. All stents and coils were delivered and released according to the standard procedures as described in the instructions of the manufacturer.

In patients in whom the luminal diameter of VBD is $>8 \mathrm{~mm}$, the diameter of the stent may not be large enough. As a result, the proximal end of the stent might swing under the blood flow, and it would be difficult to deploy the distal end of the next stent at the optimal position within the first stent. To avoid this awkward situation, before the deployment of the proximal end of the first stent at the distal normal segment of the VBD, we loosely packed a bare coil or coils in the lumen of the VBD with a double-catheter technique to support and fix the proximal end of the first stent before it was completely released; then we fully deployed the first stent. After that, the next stent was deployed with a telescoping technique. When the following stents were successfully deployed and steady apposition to the vascular wall was secured, the coil would be withdrawn to avoid occlusion of the perforating vessels from the basilar artery, as shown in our case (Fig $1 B,-C$ ). However, if the VBD was longer than the 2 stents, more coil packing was needed to fix the stents, and these could not be retrieved.

In cases in which the VBD involved bilateral vertebral arteries, we reconstructed the basilar artery and the dominant vertebral artery with stents. One month later, after confirmation that the balloon occlusion test result was negative, the nondominant vertebral artery was occluded between the basilar artery and PICA with bare coils, to reduce the blood flow into the space between the stents and the vascular wall.

Imaging and Clinical Follow-Up. With informed consent obtained from the patients, imaging follow-up including MRA at 3, 24, and 36 months and DSA at 6 and 12 months after the operation. The follow-up MRA and DSA images were compared with the immediate postembolization angiograms. Clinical follow-up by using the mRS was performed immediately after the operation; before discharge; and 1, 6, 12, 18, and 24 months after the operation. 
Clinical and imaging findings, treatment mode, and follow-up results of patients with VBD

\begin{tabular}{|c|c|c|c|c|c|c|c|c|c|}
\hline \multirow{2}{*}{$\begin{array}{l}\text { Characteristics and } \\
\text { Findings }\end{array}$} & \multicolumn{9}{|c|}{ Patient No. } \\
\hline & 1 & 2 & 3 & 4 & 5 & 6 & 7 & 8 & 9 \\
\hline Sex & $\mathrm{F}$ & $M$ & $M$ & $M$ & $M$ & $M$ & $M$ & $M$ & $\mathrm{~F}$ \\
\hline Age (yr) & 75 & 74 & 57 & 48 & 56 & 39 & 63 & 63 & 61 \\
\hline $\begin{array}{l}\text { Cerebrovascular risk } \\
\text { factors }\end{array}$ & HPN & $\mathrm{HPN}+\mathrm{HU}$ & $\mathrm{HPN}+\mathrm{HPL}+\mathrm{PK}$ & $\mathrm{HPN}+\mathrm{S}$ & $\mathrm{HPN}+\mathrm{A}$ & $S+A$ & HPN & HPN & HPN \\
\hline Symptoms & Acute headache & Ischemia & Ischemia & Acute headach & Acute headache & Ischemia & Ischemia & Chronic headache & Ischemia \\
\hline $\begin{array}{l}\text { Intraluminal } \\
\text { thrombosis }\end{array}$ & Yes (MRI) & Possible (CT) & Yes (MRI) & None & Possible (CT) & None & Yes (MRI) & Yes (MRI) & Yes (MRI) \\
\hline $\begin{array}{l}\text { Involved anterior } \\
\text { circulation }\end{array}$ & Yes & Yes & None & None & Yes & None & None & None & Yes \\
\hline Predominant VA & Yes (R) & Yes (L) & Bilateral & Yes (L) & Yes (L) & Yes (L) & Yes (R) & Yes (R) & Bilateral \\
\hline Treatment mode & $\begin{array}{c}3 \times \text { LEO, } \\
8 \times \text { coils, } \\
\text { occlude L-VA } \\
\text { I mo later }\end{array}$ & $3 \times$ LEO & $\begin{array}{l}6 \times \text { LEO, } \\
7 \times \text { coils }\end{array}$ & $5 \times$ LEO & $4 \times$ LEO & $3 \times$ LEO & $\begin{array}{c}4 \times \text { LEO } \\
1 \times \text { Solitaire }\end{array}$ & $2 \times$ LEO & $\begin{array}{c}4 \times \text { Solitaire } \\
2 \times \text { coils }\end{array}$ \\
\hline $\begin{array}{l}\text { mRS pre-op/post-op/ } \\
\text { FU (time, mo) }\end{array}$ & $\begin{array}{c}\text { 1st: } 4 / 3 / 3 \text { (1 mo); } \\
\text { 2nd: } 3 / 4 / 5 \text { (28 mo) }\end{array}$ & $\begin{array}{c}3 / 2 / 5 \\
(25 \mathrm{mo})\end{array}$ & $3 / 3 / 3(17 \mathrm{mo})$ & 1/1/0 (14 mo) & $2 / 2 / 2(18 \mathrm{mo})$ & $\begin{array}{c}\text { 3/3/Death } \\
\text { (26 mo) }\end{array}$ & $\begin{array}{l}\text { h 4/4/Death } \\
\text { (4 mo) }\end{array}$ & $1 / 1 / 0(28 \mathrm{mo})$ & 1/1/1 (10 mo) \\
\hline Angiography FU (mo) & $1 \mathrm{mo}$ & $23 \mathrm{mo}$ (MRA) & $8 \mathrm{mo}$ (DSA) & $13 \mathrm{mo}$ (DSA) & & & 8 days & $24 \mathrm{mo}(\mathrm{CTA})$ & \\
\hline
\end{tabular}

Note:-HPN indicates hypertension; HU, hyperuricemia; HPL, hyperlipidemia; PK, polycystic kidney; S, smoking; A, alcohol; L, left; R, right; pre-op, pre-operation; post-op, immediately post-operation; FU, last follow-up after the operation.

\section{RESULTS}

Nine patients were included in this study, consisting of 7 men and 2 women with a mean age of $59.56 \pm 11.45$ years (range, 39-75 years). Four of the 9 patients had anterior circulation dolichoectasia (Table). Cerebrovascular risk factors included the following: hypertension in 8 patients, hyperlipidemia in 1 patient, hyperuricemia in 1 patient, smoking history in 2 patients, alcohol consumption in 2 patients, and polycystic kidney disease with a family history in 1 patient. No diabetes mellitus, hypercysteinemia, or family history of cerebrovascular disease was elicited in these patients. The onset of disease was headache in 4 patients, and the remaining 5 patients displayed symptoms of posterior circulation ischemia, which deteriorated gradually (Table).

Of the 9 patients who underwent preoperative imaging examinations, intraluminal thrombosis or wall thrombus was found on MR imaging in 5 patients, and vertebrobasilar dolichoectasia with high-intensity shadows was found on head CT scans in 2 patients, which indicated intraluminal thrombosis or wall thrombus (Table).

Angiography showed that VBD was mainly supplied by the dominant vertebral artery in 6 of the 9 patients ( 4 by the leftdominant side). Fenestration in the basilar artery trunk was confirmed in 1 patient. One patient had subarachnoid hemorrhage due to rupture of a left posterior cerebral artery aneurysm in the P2 segment. The PCA aneurysm was embolized with sacrifice of the PCA, and the VBD was treated by multiple stent deployment 1 month after the first session (Table).

\section{Initial Results, Complications, and Clinical Outcomes}

The VBD lumen was reconstructed by using coil-assisted multiple LEO stents in 2 patients; multiple LEO stents in 5 patients; multiple LEO stents and a Solitaire stent in 1 patient; and multiple Solitaire stents and coils in 1 patient (Table). All stents and coils were placed in the intended locations as planned.

\section{Procedure-Related Complications}

One patient suddenly developed right hemiplegia on day 8 after the operation, and cerebral angiography suggested occlusion of the right anterior inferior cerebellar artery. The patient died 4 months later. One patient had VBD and bilateral ICA dolichoec- tasia, and symptoms were relieved markedly after the first VBD reconstruction. To further improve the hemodynamics of VBD, we occluded the vertebral artery on the nondominant side 1 month later. Two days after the operation, the patient developed cerebellar hemisphere and brain stem infarction, presenting with severe physical functional disability. No procedure-related complications occurred in the other patients.

\section{Clinical and Angiographic Follow-Up}

The 8 surviving patients were followed up by telephone interviews, imaging examinations, and clinical visits for a mean of $20.75 \pm 6.90$ months. One patient died suddenly at home 26 months after the operation, though no ischemic event occurred after VBD stent reconstruction during clinical follow-up. The cause of death was unknown because the family refused a postmortem examination. In 4 patients who received imaging re-examination, DSA, MRA, or CTA suggested that the vertebrobasilar artery was improved to nearly normal morphology, and vesiclelike protrusions on the arterial wall disappeared.

All patients had taken medicine according to our protocol. No patient had posterior circulation ischemic symptoms. However, 2 of 4 patients with anterior circulation dolichoectasia presented with recurrent anterior circulation cerebral infarctions. No sign or symptom of recurrence was observed in the remaining 5 patients during the $10 \sim 25$-month follow-up period.

\section{Case Illustration}

Case One. A 48-year-old male patient with a 5-year history of well-controlled hypertension and a history of excessive smoking was admitted due to a sudden onset of headache. DSA suggested VBD with hemorrhage from a ruptured aneurysm in the P2 segment of the cerebral posterior circulation and vesicle-like protrusions on the VBD arterial wall (Fig $2 A$ ). The aneurysm was tightly occluded with 6 coils (Fig 2A). Two months later, the vertebrobasilar artery was reconstructed with 5 LEO stents (Fig 2B, -C). No procedure-related complication occurred, stroke did not occur during the 14-month clinical and imaging follow-up, and the vesicle-like protrusions on the arterial wall disappeared (Fig 2D). 

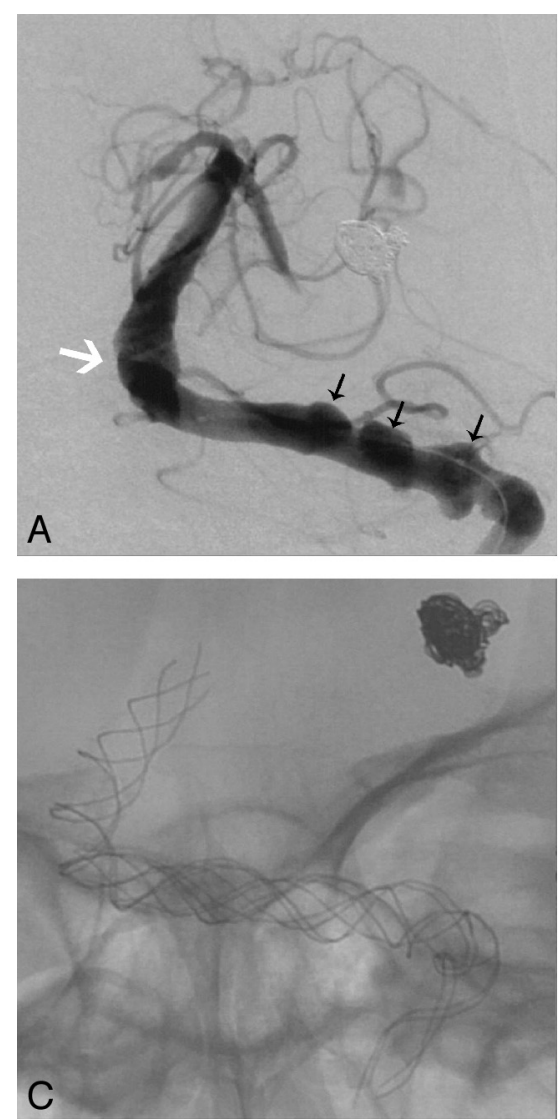

B

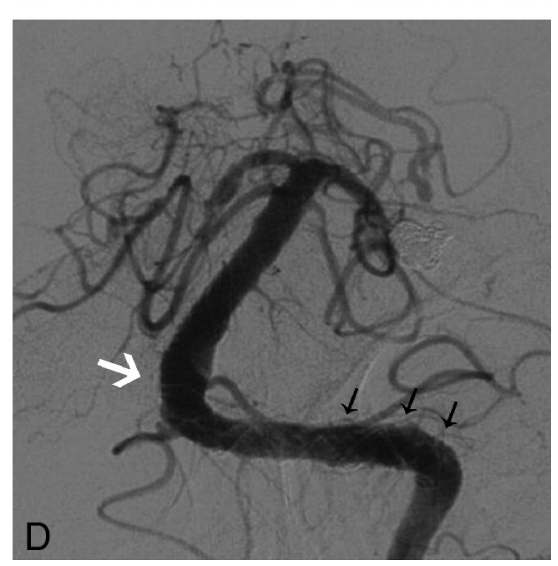

FIG 2. A, Anteroposterior position angiogram of the left vertebrobasilar artery depicts the VBD (white arrow) and aneurysmal arterial wall vesicles (black arrows). B, Angiogram immediately after overlapping stent deployment. C, Stents after the procedure. D, Angiographic follow-up 14 months after the procedure shows that the lumen of the basilar artery is thinner than that in $A$ (white arrow) and the vesicles have disappeared (black arrows).

Case Two. A 63-year-old male patient with a $>10$-year history of well-controlled hypertension was admitted because of symptoms of chronic headache and dizziness. DSA suggested a right predominant vertebral artery and VBD (Fig $1 A$ ). The vertebrobasilar artery was reconstructed with 2 LEO stents with the assistance of 1 coil, which was retrieved later (Fig $1 B$, -C). Imaging follow-up with CTA 24 months after the operation demonstrated that the fusiform aneurysm of the basilar artery had disappeared (Fig 1D).

\section{DISCUSSION}

Compared with the posterior circulation, dolichoectasia involves the arteries in the anterior circulation at a lower frequency ${ }^{9,13}$; involvement of both the vertebrobasilar and carotid systems is rare. ${ }^{14}$ In our series, 4 patients with asymptomatic dolichoectasia in the anterior circulation were followed up after endovascular reconstruction of vertebrobasilar vessels. Although the natural history of asymptomatic dolichoectasia in the anterior circulation is still unknown, clinical follow-up in this study revealed that $50 \%$ of the anterior circulation dolichoectasia could also lead to ischemic events in their territory with time, even with antiplatelet treatment after stent placement. All these findings suggest that more aggressive treatment might be needed for these patients.
The natural history of VBD usually progresses slowly, but with progression of the condition, the original symptoms may become continuously worse, posing a high risk of stroke ${ }^{6,8}$ and various adverse events. ${ }^{8}$ In addition, the death rate was significantly higher than that in patients with simple mass effect or asymptomatic VBD. ${ }^{8}$ It was found that the cumulative survival curve of patients with VBD was significantly worse than that in a control group. ${ }^{15}$ The severity of imaging presentations of patients with VBD was significantly correlated with prognosis. ${ }^{8,16}$ Follow-up observations on patients with VBD showed those patients with deteriorating imaging presentations had higher mortality and morbidity. ${ }^{8}$ In our opinion, patients with ischemic onset or physical dysfunctions or those who have deteriorating imaging presentations should be treated as soon as possible to halt disease progression.

The most common clinical manifestations of VBD are ischemia and infarction of the posterior circulation, but prevention of risk factors for stroke did not seem to have altered the occurrence of ischemic stroke significantly. ${ }^{8}$ Some researchers used anticoagulation or antiplatelet therapies to prevent infarction from occurring $^{6}$ and achieved moderate results. However, other researchers argued that the effect is not ideal in patients with VBD and may even increase the risk of intracranial hemorrhage. ${ }^{8}$

Mount and Taveras ${ }^{17}$ first reported occlusion of the vertebral artery for the treatment of VBD. Many researchers have made modifications on the basis of this method through their surgical or interventional practices ${ }^{18}$ for the treatment of VBD. However, VBD may continue to grow even though the proximal portions of the bilateral vertebral arteries have been occluded. ${ }^{19}$ This outcome suggests that the strength of the vascular wall and hemodynamics are both important factors affecting the progression of VBD. Simple reduction of blood flow may not warrant the longterm therapeutic effects.

There are cases of successful treatment of VBD by using microvascular decompression, ${ }^{20}$ Teflon (Dupont, Wilmington, Delaware) sling decompression, ${ }^{14}$ vascular clip grafting, ${ }^{21}$ synthetic vascular grafting, ${ }^{22}$ and surgical reduction of the basilar artery diameter ${ }^{23}$; but the benefits of surgery are still controversial. Moreover, all the above methods can only resolve the VBD-induced mass effect, and there is no convincing evidence to indicate that these methods can improve blood supply in the posterior circulation to prevent ischemic stroke in this territory. Some authors reported their trials with different kinds of stents and coils $^{24-30}$ to reconstruct the lumens of fusiform aneurysms of the basilar artery trunk, which may evolve from $\mathrm{VBD}^{31}$ or combine 
with VBD. However, in those articles, reconstruction of the lumen might not have been long enough to cover the VBD because their targets were fusiform aneurysms and long-term follow-up evidence was not available, either, to support the effect of ischemic stroke prevention in the posterior circulation.

In our study, we were unable to confirm the real cause of death in the patient who died suddenly 26 months after the operation due to the lack of follow-up imaging clues and postmortem examination. However, a mean of $20.00 \pm 7.09$ months' follow-up of the other 7 patients showed that multistent reconstruction in the vascular lumen for VBD could lower the occurrence of ischemic stroke in the stented vessels $(0 / 7)$ and the death rate $(0 / 7)$ compared with the natural course. In all 4 patients who had follow-up imaging data, the dilated vascular lumen became smaller and the vesicle-like protrusions on the vascular wall disappeared (Fig 1D), suggesting that stent reconstruction in the vascular lumen was successful. In addition, a mean of $20.25 \pm 8.02$ months' follow-up in the 4 patients with anterior circulation arterial dilation showed that $50 \%$ of ischemic strokes occurred in territories of untreated vessels, suggesting that stents or other more aggressive treatments are also needed for IADE involving the anterior circulation.

Some authors found that vertebral artery dominance contributes to basilar artery curvature and posterior circulation ischemic events, ${ }^{32}$ which is in line with our findings that 6 of 9 patients with VBD had a dominant VA. We suppose that modifications of hemodynamics play an important role in ischemic stroke prevention by stent deployment in patients with VBD. First, stent deployment and coil packing could change the hemodynamics and reduce the flow out of stents just like flow-diverter devices, ${ }^{29}$ which might recover the reduced axial flow. ${ }^{33}$ Second, the rigidity and elasticity of the stent can partially change the morphology of the diseased artery, ${ }^{24,34}$ thus normalizing the morphology of VBD and recovering the distortion of branches from $\mathrm{VBD},{ }^{35}$ and then increasing the flow of the branches. Third, the increased blood flow and the use of anticoagulants and antiplatelet agents after intravascular stent placement might reduce the formation of intraluminal thrombus, artery-to-artery embolism, and low-flow thrombosis in VBD. ${ }^{36}$ Stents might also reduce the risk of hemorrhage due to VBD rupture induced by the simple use of antiplatelet agents.

Vertebrobasilar artery stent placement is a safe treatment and has been widely used for the treatment of vertebrobasilar aneurysms, stenosis, and dissection owing to minimal invasiveness compared with craniotomy. In the present series of patients, we used LEO self-expanding stents and Solitaire stents. Both LEO ${ }^{37}$ and Solitaire ${ }^{38}$ stents are safe and effective devices for intravascular intervention treatment. The LEO stent can be retrieved after it has been released in $90 \%$ of cases, while the Solitaire stent is fully recoverable ${ }^{39}$ and easier to place in an intended location. ${ }^{40}$ In the present series of patients, only patients 7 and 9 had the opportunity to receive the Solitaire stent, for it was only available in Changhai Hospital from September 2010.

The main procedure-related complication was postoperative occlusion of the branches, amounting to $22.2 \%$ in total. This high occurrence may be attributed to embolus formation due to flow retardation between the stent and the vascular wall due to altera- tion of the hemodynamics induced by the stent struts and coils. Therefore, a more suitable attenuation of stent mesh is needed. We suppose that multilevel and staged stent placement may be a safer strategy - that is, first covering the diseased vessel by a telescoping technique (Fig 2C) with multiple stents in a single layer and minimizing the coil implantation and then implanting the stents again in 3 months. After that, more stent implantations may be needed according to angiographic follow-ups until the location of hemodynamic insufficiency is confirmed so as to gradually change the hemodynamics in VBD to reduce the occurrence of complications.

\section{Limitations of the Study}

Because the incidence of VBD is relatively low and the number of VBD cases that we have treated is not large enough, studies with more cases and longer follow-up are needed to evaluate the longterm effect of this method in preventing stroke. In cases in which VBD involves bilateral vertebral arteries, the necessity to occlude the nonstented vertebral artery for further alteration of hemodynamics needs to be further studied. We are convinced that with more understanding of hemodynamic changes in individual patients, the dilemma of hypo- and hyperhemodynamics arising from stent placement can be solved. Further studies are also needed to explore whether IADE involving the anterior and posterior circulation should be managed simultaneously and which therapeutic strategy should be used in such cases.

\section{CONCLUSIONS}

Endovascular reconstruction by using coil-assisted stent placement techniques or stent placement alone in the vascular lumen for the treatment of VBD is technically feasible and safe. It could prevent ischemic events in stented vessels compared with the natural disease course, though further studies in larger samples are needed.

\section{ACKNOWLEDGMENTS}

The authors would like to acknowledge the invaluable contributions of He Ying. This study would not have been possible without her support.

\section{REFERENCES}

1. Yu YL, Moseley IF, Pullicino P, et al. The clinical picture of ectasia of the intracerebral arteries. J Neurol Neurosurg Psychiatry 1982;45:29-36

2. Pico F, Labreuche J, Cohen A, et al, for the GENIC investigators. Intracranial arterial dolichoectasia is associated with enlarged descending thoracic aorta. Neurology 2004;63:2016-21

3. Resta M, Gentile MA, Di Cuonzo F, et al. Clinical-angiographic correlations in 132 patients with megadolichovertebrobasilar anomaly. Neuroradiology 1984;26:213-16

4. Ince B, Petty GW, Brown RD Jr, et al. Dolichoectasia of the intracranial arteries in patients with first ischemic stroke: a populationbased study. Neurology 1998;50:1694-98

5. Dziewasa R, Freund M, Lüdemann P, et al. Treatment options in vertebrobasilar dolichoectasia: case report and review of the literature. Eur Neurol 2003;49:245-47

6. Wolfe T, Ubogu EE, Fernandes-Filho JA, et al. Predictors of clinical outcome and mortality in vertebrobasilar dolichoectasia diagnosed by magnetic resonance angiography. J Stroke Cerebrovasc Dis 2008;17:388-93

AJNR Am J Neuroradiol 34:583-88 Mar 2013 www.ajnr.org 
7. Pico F, Labreuche J, Touboul PJ, et al. GENIC Investigators. Intracranial arterial dolichoectasia and its relation with atherosclerosis and stroke subtype. Neurology 2003;61:1736-42

8. Passero SG, Rossi S. Natural history of vertebrobasilar dolichoectasia. Neurology 2008;70:66-72

9. Gutierrez J, Sacco RL, Wright CB. Dolichoectasia: an evolving arterial disease. Nat Rev Neurol 2011;7:41-50

10. Smoker WR, Price MJ, Keyes WD, et al. High-resolution computed tomography of the basilar artery. 1. Normal size and position. AJNR Am J Neuroradiol 1986; 7:55-60

11. Giang DW, Perlin SJ, Monajati A, et al. Vertebrobasilar dolichoectasia: assessment using MR. Neuroradiology 1988;30: $518-23$

12. Jeng JS, Yip PK. Evaluation of vertebral artery hypoplasia and asymmetry by color-coded duplex ultrasonography. Ultrasound Med Biol 2004;30:605-09

13. Lou M, Caplan LR. Vertebrobasilar dilatative arteriopathy (dolichoectasia). Ann N Y Acad Sci 2010;1184:121-33

14. Takeuchi S, Takasato $\mathrm{Y}$, Masaoka H, et al. Dolichoectasia involving the vertebrobasilar and carotid artery systems. J Clin Neurosci 2009; 16:1344-46

15. Ubogu EE, Chase CM, Verrees MA, et al. Cervicomedullary junction compression caused by vertebral artery dolichoectasia and requiring surgical treatment: case report. J Neurosurg 2002;96:140-43

16. Pico F, Labreuche J, Gourfinkel-An I, et al. GENIC Investigators: basilar artery diameter and 5-year mortality in patients with stroke. Stroke 2006;37:2342-47

17. Mount LA, Taveras JM. Ligation of basilar artery in treatment of an aneurysm at the basilar-artery bifurcation. J Neurosurg 1962;19:167-70

18. Chao KH, Riina HA, Heier L, et al. Endovascular management of dolichoectasia of the posterior cerebral artery report. AJNR Am J Neuroradiol 2004;25:1790-91

19. O'Shaughnessy BA, Getch CC, Bendok BR, et al. Progressive growth of a giant dolichoectatic vertebrobasilar artery aneurysm after complete Hunterian occlusion of the posterior circulation: case report. Neurosurgery 2004;55:1223

20. Pereira-Filho A, Faria M, Bleil C, et al. Brainstem compression syndrome caused by vertebrobasilar dolichoectasia: microvascular repositioning technique. Arq Neuropsiquiatr 2008;66:408-11

21. Laws ER Jr, Kelly PJ, Sundt TM Jr. Clip-grafts in microvascular decompression of the posterior fossa. Technical note. J Neurosurg 1986;64:679-81

22. Ogawa A, Suzuki M, Shirane R, et al. Repositioning of the tortuous vertebrobasilar artery for trigeminal neuralgia: a technical note. Surg Neurol 1992;38:232-35

23. Matsumoto K, Yamada K, Hayakawa T, et al. Dolichoectatic basilar artery treated by reducing hemodynamic stress: report of two cases. Neurol Med Chir (Tokyo) 1990;30:691-94

24. Crowley RW, Evans AJ, Kassell NF, et al. Endovascular treatment of a fusiform basilar artery aneurysm using multiple "in-stent stents": technical note. J Neurosurg Pediatr 2009;3:496-500
25. Fiorella D, Albuquerque FC, Han P, et al. Preliminary experience using the Neuroform stent for the treatment of cerebral aneurysms. Neurosurgery 2004;54:6-16, discussion 16-17

26. Zenteno MA, Murillo-Bonilla LM, Guinto G, et al. Sole stenting bypass for the treatment of vertebral artery aneurysms: technical case report. Neurosurgery 2005;57(1 suppl):E208, discussion E208

27. Hassan T, Ezura M, Takahashi A. Treatment of giant fusiform aneurysms of the basilar trunk with intra-aneurysmal and basilar artery coil embolization. Surg Neurol 2004;62:455-62, discussion 462

28. Binning MJ, Natarajan SK, Bulsara KR, et al. SILK flow-diverting device for intracranial aneurysms. World Neurosurg 2011;76: 477.e1-6

29. Bain M, Hussain MS, Spiotta A, et al. "Double-barrel" stent reconstruction of a symptomatic fusiform basilar artery aneurysm: case report. Neurosurgery 2011;68:E1491-96, discussion E1496

30. Higashida RT, Smith W, Gress D, et al. Intravascular stent and endovascular coil placement for a ruptured fusiform aneurysm of the basilar artery: case report and review of the literature. J Neurosurg 1997;87:944-49

31. Bezerra S, Casaroto E, Bueno Alves M, et al. The challenge of managing fusiform basilar artery aneurysms: from acute ischemic stroke to a massive subarachnoid hemorrhage. Case Rep Neurol 2011;3:50-53

32. Hong JM, Chung CS, Bang OY, et al. Vertebral artery dominance contributes to basilar artery curvature and peri-vertebrobasilar junctional infarcts. J Neurol Neurosurg Psychiatry 2009;80:1087-92

33. Amin-Hanjani S, Du X, Zhao M, et al. Use of quantitative magnetic resonance angiography to stratify stroke risk in symptomatic vertebrobasilar disease. Stroke 2005;36:1140-45

34. Huang QH, Wu YF, Xu Y, et al. Vascular geometry change because of endovascular stent placement for anterior communicating artery aneurysms. AJNR Am J Neuroradiol 2011;32:1721-25

35. Passero S, Filosomi G. Posterior circulation infarcts in patients with vertebrobasilar dolichoectasia. Stroke 1998;29:653-59

36. Kumral E, Kisabay A, Ataç C, et al. The mechanism of ischemic stroke in patients with dolichoectatic basilar artery. Eur J Neurol 2005; 12:437-44

37. Kis B, Weber W, Götz F, et al. Endovascular treatment of cerebral aneurysms using the LEO stent: long-term follow-up and expansion of indications. Clin Neuroradiol 2007;17:167-79

38. Klisch J, Eger C, Sychra V, et al. Stent-assisted coil embolization of posterior circulation aneurysms using Solitaire AB: preliminary experience. Neurosurgery 2009;65:258-66, discussion 266

39. Papanagiotou $P$, Roth C, Walter S, et al. Treatment of acute cerebral artery occlusion with a fully recoverable intracranial stent: a new technique. Circulation 2010;121:2605-06

40. Krischek O, Miloslavski E, Fischer S, et al. A comparison of functional and physical properties of self-expanding intracranial stents [Neuroform3, Wingspan, Solitaire, Leo +, Enterprise]. Minim Invasive Neurosurg 2011;54:21-28 